\title{
Uwe Wesel Zur Entstehung von Recht in frühen Gesellschaften
}

Irgendwann ist Recht entstanden. Die Neandertaler jedenfalls haten es noch nicht entdeckt. Es gibr kein diluviales Recht der Eiszeit. Und in Rom, bei der Abfassung des Zwölfrafelgesetzes, am Beginn der Republik, existierte es schon auf der Stufe der Kodifikarion. In der Zwischenzeit muß geschehen sein, worüber Juristen bisher kaum nachgedacht haben. Für Savigny war Recht das Ergebnis des stillen Wirkens eines Weltgeistes. Henry Maine meinte, es sei aus der Religion entstanden. Der religiöse Ursprung ist eine sehr alte Behauptung, mit der schon große Kodifikationen der Ancike sich schmückten. Allgemein lebt man heure im beruhigenden Gefühl, mit dem Recht sei es schon immer so gewesen, und was wir vor uns haben nur das selbstverständliche Ereignis seiner ewigen Wiederkehr. Die historische Frage der Entstehung wird nicht gestellt.

\section{Was ist Recht?}

Die Rechrsgeschichte konnte einer ernsthaften Beantwortung dieser Frage bisher immer ausweichen, weil sie sich nur in Bereichen bewegte, in denen man die Existenz von Recht voraussezzen durfte. Anders die Ethnologie. Sie bewegt sich auch in anderen. Seir Jahrzehnten wird hier über die Abgrenzung von law und custom, von Recht und Gewohnheit, diskutiert. Selbstuerscändlich gibt es in allen trühen Geselischaften Regeln für das Zusammenleben der Menschen, auch bei den Jägern. Aber ist jede Regel Recht, wie manche arnehmen? Oder muß man unterscheiden, je nachdem ob es Gerichte gibt? Die meisten stellen es auf die Möglichkeit physischer Sanktionen ab, einige nehmen an, Recht sei erst dann vorhanden, wenn beides gegeben ist, Gerichte und die Möglichkeit physischer Sankrionen. Heute ist man sich jedenfalls danüber einig, daß es sehr viele Gesellschaften gibr, die ohne Rechr, nur mit Gewohnheicen leben. Aber die Abgrenzung im einzelnen, meinen die Ethnologen, sei eines der schwierigsten Probleme ihrer Arbeit. Es gäbe keines, auf dessen Klärung sie so viel Mühe verwendet hätten. Ihre Schwierigkeiten werden verstärkı durch Mißverständnisse über Rechtstheorien der Juristen. Die juristischen Theorien zur Abgrenzung zwischen Recht und Gewohnheit sind nämlich entwickelt für Gesellschakten, in denen es selbstverständlich immer auch Recht gibr. Juristen können sich nicht vorstellen, daß Gesellschaften ohne Recht exiscieren. Es geht bei ihnen nur um Abgrenzungen eines internen Nebereinanders. Die Ethnologen suchen nach einer Abgrenzung zwischen Rechtsgesellschafien und Gewohnheirsgesellschaften.

Der Einwand liege nahe, das Ganze sei doch nur ein Streit um Worte. Warum soll man nicht einfach jede gesellschaftiche Norm als Rechs bezeichnen? Nun, die Echnologen haben einen anderen Blick als wir, einen besseren. Besonders die 
englische Schule, aber auch die amerikanische, interessier sich in erster Linie dafür, wie diese Gesellschatten funktionieren. Gewohnheitsgesellschaften funkrionieren anders als Rechtsgesellschaften, nämlich selbstätig, selbstregulierend. Rechtsgesellschaften werden gesteuert. Regel ist nicht gleich Regel. Es kommt auch daraut an, wie sie funkcioniert, wie sie gehandhabc wird, wie sie entsteht, sich verändert, welchen Inhalt sie bat. Es ist die Frage des Funktionierens verschiedener Regeln in verschiedenen Gesellschaften. Im übrigen wird sjch zeigen, bei der Beschreibung der Gesellschaft der Aschanti in Westafrika, daß sie selbst diese Unterscheidung machen. Sie sind eine rypische Ubergangsgesellschaft, eine Rechtsgesellschaft mit Resten einer Gewohnheitsgesellschaf. Sie sprechen nicht abstrakt von "Regein", sondern von efiesem, das abgeleiter ist von fie, das Haus. Das ist die Ordnung der Gewohnheiten. Und sie sprechen von oman akyiewadie, das ist die Herrschaft des Rechis.

Die Ethnologen haben bei der Abgrenzung den Fehler gemacht, sich an die formalen Rechtstheorien der Juristen zu halren. Es sind formale Rechrscheorien, die im Grunde auch nur für die bürgerliche Gesellschaft passen. Man muß startessen versuchen, die Begriffe aus der Strukcur und der Encwicklung dieser Gesellschaften abzuleiten und auszufüllen. Den richtigen Weg dorthin hat Stanley Diamond gewiesen. Das Ergebnis, das hier vorweggenommen wird, weil der encwickelte Begriff den verwickelten Weg besser erkennen läßt, ist das folgende.

Frühe Gesellschafen sind egalitär, in einem sehr bewußten Sinn. Irgendwann, in regelwidriger Störung ihrer egalizären Ordnung, entsteht Ungleichheic aus dieser Gleichheit, entsteht Herrschaft, mit einer neven Ordnung, die die alte grundlegend

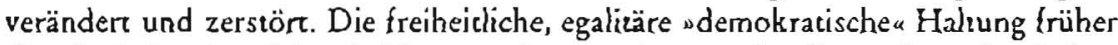
Gesellschaften ist oft beschrieben worden, von Morgan für die nordamerikanischen Indianer mit ihrer "Freiheit, Gleichheit und Brüderlichkeit" als Grundprinzipien der Gens, bis zu Clastres, der die südamerikanischen Indianer als entschiedene Staarsfeinde bezeichnet hat, als Feinde von Staarlichkeit. Die Lele, Ackerbauern im westlichen Kongo, sagen von sich selbst, sie seien sehr neidisch und dulderen keine Ungleichheit. Evans-Pritchard sprichr vom egalieären Bewußssein der Nuer, einem Hirtenvolk im südlichen Sudan, das cief demokratisch ist: They strut about like Lords of the earth. Die Mbuti-Jäger des Regenwaldes am Uturi im rördilichen Kongo machen sich lustig über die angrenzenden Dorfbewohner, die selbstverständlich davon ausgehen, bei ihnen gäbe es in jeder Horde einen Anführer. Jede Tendenz zu charismatischem Führertum verfällc bei ihnen der Lächerlichkeir. Die Mechanismen zur Verhinderung der Entstehung von Ungleichheit und Herrschaft sind stark. Trotzdem gibt es ab und zu Durchbrüche, wird der Weg Irei zur Kephalizär, zur Veränderung der gesamten Strukrur. Frühe Ackerbaugesellschafren sind segmentär organisier, bestehen aus einem Nebeneinander autonomer Verwandtschaftsgruppen, die gemeinsam, Segment an Segment, die Gesellschaft bilden, ohne Häuptlinge oder Könige. Das läßr sich in der Geschichte und in der Ethnologie beobachten. Die Egalität ihrer Ordnung beruht auch auf dem gleichberechrigren Nebeneinander dieser Gruppen. Gesellschaften mic Zentralinstanzen, Häuptlingsgesellschaften, frühe Königreiche, Protostaazen, sind in der Geschichce wie in der Echnologie die nächste Stufe. Man nennt sie kephale, vom griechischen kephalos, der Kopl. Ihre Entstehung ist nicht nur ein Problem für Ethnologen, es ist auch eines im Bewußtsein der frühen Gesellschaften. Wie wird Entstehung von Herrschaft verhindert? Wie entsceht sie trotzdem? Wie entsceht Kephalität aus Akephalizät, Ungleichheir aus Gleichheit, Herrschaf́r aus Freiheir? Hier nämlich kommen wir auch aul den Begriff des Reches. Recht entsceht mic Herrschaft im Ubergang von segmentären zu kephalen Gesellschafren. Gewohnheit und Rechr 
sind nicht, wie Henry Maine meinte, kontinuierliche Formen von im übrigen inhaltsgleichen Regeln, also in einer Reihe zu sehen als Entwicklung von custom über customary law zu law. Sie sind Gegensätze. Sie haben nicht nur verschiedene Gelrungskraft, sondern auch verschiedene Grundlagen, Bedingungen und verschiedene Inhalte. Recht ist nicht die kontinuierliche Weiterentwicklung von Gewohnheiten der alten Ordnung, sondern ihre Zerstörung, die verbunden ist mit dem allmählichen Entstehen einer neuen. Auf dem Weg von der Gewohnheirsgesellschaft zur Rechusgesellschaft gibr es Zwischenbereiche, die man als jural bezeichnen kann, wie Meyer Fortes es tur. Rechr ist nicht nur ein qualitacives, es ist auch ein quantitatives Element menschlicher Existenz. Es entsteht, breiter sich aus, überlagert, absorbiert und zerstört schließlich die alte Ordnung der Gewohrheiren, enfaßr ständig neue Bereiche gesellschafclicher Ordnung und kann im Extremfall das ganze Leben der Menschen bestimmen, wie in der bürgerlichen Gesellschaft, die cotal verrechslicht worden ist. Custom dagegen, Gevohnheit, die alte egalitäre Ordnung, ist stacisch, eher friedlich, selbstregulierend, konsensual auf Einigung gerichtec, niche nur auf die Einigung zweier Parteien, sondern auf den kollektiven Konsens aller, Gemeinschaftlichkeic. Die Einigung wird, wenn es Konflikte gibt, durch das Gespräch erreicht, das solange geführe wird, bis alle überzeugt sind. Auch hier gibr es Gewalt, physische Gewalt. Aber sie ist individuell, ungeordnet, wild und wird von innen heraus durch die Ordnung des Gesprächs und gemeinschaftlichen Konsenses überwunden. Es ist die Ordnung der Jäger und der segmentären Gesellschaften, sich selbst regulierende Anarchie. Recht dagegen ist die Uberlagerung, Absorprion und Zerstörung von Gewohnheit durch zentrale Herrschaft, die es zunehmend und bewruß als Instrumentarium von außen einsetzt, ist steuerbar, verfügbar und verbunden mit physischer Sankcion, also apparative Gervalt, während Gewohnheit das kollektive Gespräch war. Gleichzeitig ist das Recht individualisierend, weil herrschafrliche Gewalt sich zuerst gegen einzelne richcet. Seine Verfügbarkeit bedeutec, daß es stärker auf Veränderung, auf Geschichte zielt. Die Geschichte der Entstehung von Recht ist also auch die Entstehung von Herrscbaft als zwangsausübender Machr. Sie ist die Geschichte der Kephalität, der Entstehung von Ungleichheit und der Zerstörung einer egaliären und freiheitlichen Ordnung.

\section{Mitgliedschaft und Flukewation in Jägergesellschaften}

Von den zwei Millionen Jahren ihrer Existenz haben die Menschen nur etwa ro 000 Jahre nicht als Jäger gelebt. Ein halbes Prozent der Zeit. Es war die erste Uberflußgesellschaft, first affluent society, wie Marshall Sahlins sie genannt hat. Die Menschen arbeiten zwei bis vier Stunden täglich. Viel Müßiggang gibt es, viel Schlaf, auch am Tage. Zeitweise auch Hunger, aber nicht so viel wie heute. Sie sind sehr fröhlich, produzieren und konsumieren für den Tag, haben keine Vorstellung von Zeir und planen niche für die Zukunft wie Ackerbauern. Die Abhängigkeit vom Jagdglück isc nicht so groß, wie man gewöhnlich meint, denn Fleisch macht nur 20-40\% ihrer Diät aus. Der Rest ist pflanzliche Nahrung, sind Beeren, Wurzeln, Blätcer, Lianen, Nüsse. Sie leben in Horden von zwanzig bis fünfzig Personen. Es gibr keine feste Verwandtschaftsstnktur wie bei frühen Ackerbauern. Die Fluktuation zwischen den Horden ist sehr groß. Die Horde ist aufgebaut auf Mitgliedschaft, nicht auf Verwandischaft. Mirgliedschaft bedeuter einfach akzeptierte Zugehörigkeit, möglichernveise seit der Geburt, aber auch durch Zugang von außen. Das kollektive Unternehmen der Jagd endet täglich mit der Verteilung des Produkts unter die Produzenten. Es wird gleich konsumier, nicht konserviert oder akkumu- 
liert. Weil eine Akkumulation nicht scarffinder, können sic sich das labile Gleichgewicht ihrer Horde mit der jederzeit möglichen Lösung des Weggangs einzelner bei schwereren Konflikten leiscen, denn der Weggang bringz weder Teilungs- noch Produkrionsprobleme. Die Flukruation ist die Existenzgrundlage der Horde in einem doppelren Sinn. Sie sorgt durch Zugang oder Weggang für dic ökologisch richrige Größe der Horde und dient gleichzeitig als äußerstes Mittel der Konflikclösung zur Erhaltung des gesellschafulichen Gleichgewichts.

Es gibt eine Arbeitsteilung der Geschlechter. Die Frauen sammeln, die Männer jagen: die erste große Arbeitsteilung, nicht die in Ackerbau und Viehzucht, wie Engels noch meinte. Sie führt über das hohe Sozialprestige des Jägers zu einem leichten gesellschaftlichen Übergewicht des Mannes über die Frau. Die Gründe für die sexuelle Arbeitsteilung in Jägergesellschaften sind noch nicht geklärt, also ob sie gesellschaftlicher oder biologischer Natur sind. Jedenfalls führt sie im allgemeinen nicht zu einer starken Benachreiligung der Frauen, auch insofern sind Jäger egalï̈r. Die Arbeir spielt bei ihnen keine so große Rolle wie später im Ackerbau. Deshalb hat ihre Teilung auch noch keine größeren Folgen. Ibre Egalicär beruht im übrigen auch auf dem Mangel an Habe, die eine Last wäre auf ihren Wanderungen von einem Lager zum anderen, das sie etwa alle zwei bis drei Wochen wechseln. Jäger begnügen sich mic einem Minimum an Gerätschaften. Konflikte darüber gibt es kaum. Sie kennen kein Eigenrum.

Mangel an strukturellem Druck kennzeichner auch die Familie. Eine Frau und ein Mann ziehen einfach zusammen, ohne Hochzeitsriten, leben in einer Hüre. Das ist alles. Ebenso leicht geht man wieder auseinander. Im Laufe der Zeit wird die Verbindung fester, besonders wenn Kinder da sind. Die Erzeugung von Kindern ist nicht gesellschaftlicher Zweck der Verbindung, weil die Horde ihre Lebensfähigkeit auch durch Zugang von außen aufrechterhalten kann. Also ist es eine sehr freie Verbindung von Frau und Mann, auf die noch kein gesellschafulicher Druck ausgeübr wird. Dementsprechend gibc es keinen Ahnenkule, keine Religion, nur pantheistische Vorstellungen, regelmäßig vom Wald bescimmt, und einfachen Geisterglauben, der keine gesellschaftliche Kraft darstellt wie Zauberei und Hexerei bei frühen Ackerbauern. Auch Jagdzauber spielt keine große Rolle. Tore werden unauffällig begraben, häufig einfach die Hürte über ihnen abgerissen und das Lager verlassen.

Das Kollektiv der Horde bestimme sich selbst. Entscheidungen über die Jagd, Abbruch des Lagers und den Ort des nächsten werden gemeinschafulich getrolfen. Einzelne haben größere Autorität, besonders die erfolgreichen Jäger, aber sie müssen sich zurïckhalken, sind immer, wenn sie es nicht tun, in Gefahr der Lächerlichkeit, und können jederzejt überstimmt werden. In einigen Jägergesellschaften gibr es Anführer der Horden, in anderen nicht. Guayaki und Eskimo z. B. kennen Sprecber, aber sie befinden sich auch in Sondersituationen, in einer feindlichen Umwelt oder ökologisch extremen Umgebung. Die Mbuti, die eher typisch sind für historisch frühe Jäger, haben keine. Aber auch bei den Guayaki erhielt Clascres auf die Frage, was der Anführer der Gruppe zu tun habe, die Antwort: „Er tut überhaupt nichts, er ist derjenige der gewöhnlich spricht.. Ebenso bei den Eskimo. Der Sprecher ihrer Siedlung wird nicht ausdrücklich gewählt, nur mehr oder weniger stillschweigend́ anerkannt und kann auch jederzeic wieder aus der Siedlung herausgedrängt werden, wenn er sich nicht gruppenkonform verhäl. Er ist nur der, „dem alle zuhören $\%$. Das entspricht dem, was Clastres allgemein als Kennzeichen südamerikanischer Indianer beschrieben hat, der absolucen Negacion von Machc. Die Gruppe behäle ihre eigene Souveränität. Jäger sind anarchisch, herrschaftufrei. 
Der anarchische Charakter der Horde finder sich wieder in den Mechanismen der

Konfliktlösung. Auch bei Jägern gibt es Konflikte, die das Gleichgewich der Gruppe gefährden und bei uns die Frage des Rechts stellen würden. Meistens geht es um die Jagd, um die Faulheit einzelner z. B., die zum Mißerfolg führt, der Folgen für alle hat, wenn das Produkt geteilt wird. Oder es geht um Lärm, auch von Kindern, der das Wild vertreibt, um die Verteilung der Beute, für die es fesce Regeln gibt, die verletzt werden können. Alle Streitigkeiten werden beigelegt in gemeinsamer Diskussion, kleine in kleinem Kreise, größere mit der ganzen Horde. Die Älteren schlichcen, und zwar Männer ebenso wie Frauen. Man redet so lange, bis eine Einigung erreicht ist. Nur selten gibc es dabei eine feste Entscheidung. Norfalls verläßt einer die Gruppe und wechselt zu einer anderen Horde, manchmal auch mehrere. Die Flukeuacion hat die Funkcion der Autrechterhalung des sozialen Gleichgewichts, etwa bei Streitigkeiten aus Eifersucht. Sanktionen gibe es kaum, schon gar keine festen Regeln. Allenfalls gibe es einmal einen erregten Ausbruch einiger Mirglieder der Gruppe gegen einen anderen. Tumbull beschreibr, wie bei den Mburi einem läger, der sein Necz bei der Treibjagd vor dem Halbkreis der Gruppe aufgestell, hatte, die Hütte niedergerissen wurde.

Das ist die Ordnung der jäger, aufgebaut auf der Mirgliedschaft in der Horde. Die Gesellschaft ist bewußt egalizär. Das Kollektiv der Horde bestimmr sich selbst. Das soziale Gleichgewicht erhält sich selbstregulierend im Konsens aller. Konflikte werden gelöst im gemeinschaftlichen Gespräch oder durch Fluktuation, die auch die ökonomisch richtige Größe der Gruppe regulierr. Es gibt keine Herrschaft und kein Recht. Die Gesellschaft ist anarctuisch und friedlich. So hat man bis zum Ende der Alcsteinzeit gelebr.

\section{Selbstregulierende Ordnung in segmentären Gesellschaften}

Mit der Jungsceinzeic geht man allmählich zur Seßhafrigkeit über, um das 9. Jahrtausend v. Chr., in Nordafrika, Mesoporamien, Nordsyrien, Südanacolien, vom food gathering zum food producing. Gordon Childe hac es als die neolithische Revolution bezeichnet. Das Ende der leczten Eiszeit bedeutete, mit dem Rückgang der Vereisung im Norden, eine scarke Austrocknung dieser südlichen Gebiete, in denen die Jagd damic schwierig wurde. Es entsteht zuerss eine gemischte Landwirtschaft, Ackerbau mit Kleinviehzucht, kombiniert mit Jagd. Die Landwirtschaft bringt die Möglichkeic für die Konservierung der Produkte und Akkumulation von Surplus. Es ist eine Hauswirtschaft mit Töpferei und Weberei. Die Grundlagen werden geschaffen für die erscaunliche Entwicklung der Produkcivbräfte in den Hochkulturen Ägyprens und Mesopocamiens. Die Zunahme der Bevölkerung ist sehr groß. Alles deuter darauf hin, daß die Gesellschaft von Ansang an segmentär organisiert war. Eindrucksvoll deutlich wird dies in der Struktur der um rg60 von dem englischen Archäologen James Mellaart ausgegrabenen Siedlung Caral Hüyük aus dem frühen sechsten Jahrtausend in Südanatolien: Jeweils mehrere Wohnhäuser liegen um eine gemeinsame Kultstätte für die gemeinsamen Ahnen. Im äußersten Oscen Mesopotamiens ist es in Keilschriftexten nachgewiesen, ebenso wie noch für das letzte Jahrtausend vor Christus bei den Lykiern im Westen Anatoliens.

Diese segmencären Gesellschaften sind im Gegensatz zu Jägern stärker strukturiert und klar geglieder. Die Gliederung wird hergestelt über die Ordnung der Verwandischaft. Während Jägergesellschaften nur die lockere Bindung der Horde kennen, entsteht mic der Seßhafrigkeir das feste System der Verwandschafr, entstehc Verwandtschaít als gesellschafuliche Realität. Das war die große Encdeckung Mor- 
gans bei den Irokesen, die Identität von Verwandischaftssystem und golicischer Ordnung, die in einem Nebeneinander besteht von agnarischen Verwandischaftsgruppen, die als selbständige und in sich geschlossene Segmence der Gesellschaft gemeinsam einen Stamm bilden, dessen gesellschafticher Zusammenhalt hergestellt wird durch die Exogamie der Segmente und die Endogamie des Stammes, also durch die Regel, daß man nur außerhalb der agnacischen Verwandischaftsgruppe, aber innerhalb des Stammes heiraten darf. Im Gegensatz zum ungegliederten kognacischen Verwandischafissystem, der Blurverwandzschaft, die auch unserer Vorstellung entsprichr, zählt im agnarischen nur die Verwandtschaft über die Murter - Matrilinearirät - oder die über den Vater - Patrilinearität. Ein Kind im kognatischen System ist zweilinig verwandt mit der Familie der Mutter und des Vaters, im agnarischen System einlinig, entweder nur mit der Familie der Mutter oder der des Vaters. Durch diese einlinige Gliederung entstehen feste Verwandtschaftsgruppen, Deszendenzgruppen, die man als gens, lineages, kin group oder clan bezeichner. Innerhalb dieser Gruppen darf man nicht heiraten, und zur Verstärkung des Exogamiegebots gibc es regelmäßig das Inzestrabu.

Die lineages sind verhälenismäßig groß, zählen fünf bis acht Generauionen zurück und haben in der Regel einen Stammsitz in einem Dorf, das ihnen "gehöru. Ihr Nebeneinander in frühen Gesellschaften, die Abwesenheit einer staaclichen Zentralinstanz, der dadurch gewährleiscete Raum für Freiheit, das war die Encdeckung Henry Morgans, die dann ausgebaut worden ist von der englischen social anthropology, von Radcliffe-Brown und seinen Schülern.

Man kennt heute hunderte solcher Gesellschafien, überall in der außereuropäischen Welt, in Amerika, Afrika, in Asien und in der Südsee. Sie sind von der social anthropology als akephale abgegrenzt worden gegenüber kephalen Häuptlingsgesellschaften und frühen Königreichen. Es gibr Ubbengangsgesellschaften mir Kephalität und starker segmentären Grundstruktur, wie z. B. die der Aschanti in Ghana. Die historisch frühen sind immer die akephalen, segmentären. Sie sollen hier besctrrieben werden am Beispiel der Nuer, Tallensi und Lele. Die Nuer sind ein patrilineares Hirtenvolk im südlichen Sudan, die Tallensi leben als patrilineare Ackerbauern im südlichen Obervolta an der Grenze zu Ghana, und die Lele sind matrilineare Ackerbauern am Kasai, einem südlichen Nebenfluß des Kongo. Sie sind erforscht worden von Evans-Pricchard, Fortes und, zuletze die Lele, von Mary Douglas. Es sind Gesellschaften ohne Staat, ohne Zentralinstanz, ohne Herrschaft. Ihr Gleichgewicht erhält sich durcl das selbscändige Nebeneinander ihrer Segmente, der lineages. Diese Segmente sind in ständiger Veränderung, sie wachsen, teilen sich, neue Segmente entstehen, aber sie scellen sich doch auch wieder dar in einer Stabilität, die jedenfalls, zunehmend mit der Konsistenz der Produktionsmitcel, immer sehr viel größer ist als die der Horden in Jägergesellschaften. Am geringsten ist sie in Hackbaugesellschafcen mit Jagd (Lele), am größten in Gecreidebau- und Hirtengesellschaften (Tallensi, Nuer). Je stärker in ihnen Stabilität und Seßhafrigkeit sich ausbilden, desto größer werden auch die Autorität der Älteren, ihre Kompecenzen und desto intensiver wird der Ahnenkult. Die lineage ist keine Gebietseinheit, ebensowenig wie das Dort. Sie ist personell, dynamisch und segmentär. Nicht das Land gibt den Zugang zu den Produkzionsmitteln, sondern die lineage, die nicht ein territoriales, sondern soziales Gebilde ist. Produkcionseinheit und Konsumprionseinheit ist der Hof, die nnuclear lineage ${ }^{\prime}$, auf der in der Regel ein Mann mit seiner Frau lebr, mit seinen Eltern häufig, und mir seinen Brüdern und Kindern. Innerhalb der lineage und des Dorfes sind alle gleich. Es gibe Alteste, Sprecher, den Dorfätesten und einen Sprecher bei den Lele, einen dominierenden Mann in jedem. Dorf, der von den Engländern als «bull $\ltimes$ bezeichnet wird, und die Leopardenfell-Priester bei 
den Nuer, einen lineage-Ältesten und einen Erdpriester bei den Tallensi. Sie haben Autorität, einen gewissen Einfluß, aber keine Macht und sind abhängig vom Konsens der Gemeinschaft. Die Leopardenfell-Priester der Nuer zum Beispiel sind in ihrer wichtigsten Funktion, der Beilegung von Blutfehden, aut eine unverbindliche Vermirclerrolle beschränkt, angewiesen auf ihr persönliches Geschick und den Willen der bereiligten lineages zur friedlichen Beilegung.

Eigentum verstehen sie weitgehend in den Begriffen von Verwandrschaft. Es ist Kollekciveigentum. Für Veräußerungen braucht man die Zustimmung der anderen. Wenn das Land knapp ist, wie bei den Tallensi, die sehr dicht siedeln, dann gibe es auch Eigencum daran, weniger bei den Lele und Nuer. Veräußerungen gibs es nichn nur die Vererbung. Bei den Tallensi uncerscheider man allgemein zwischen Erbgut und Individualgut. Individualgut ist das, was jemand bei Lebzeicen selbst erworben hat. Bei seinem Tode verwandeit sich auch dieses Individualgut in Erbgut. Die Teilung von Erbgut ist selten. Beim Tode des Nuer-Vaters wird die Herde nicht geteilt. Die Brüder übernehmen sie mic gleichen Rechren. Rinder haben bei ihnen außer ihrem ökonomischen Zweck auch noch im wesentichen die Funkrion als Brautpreis. Das ist das wichtigste Eigentumsproblem in frühen Gesellschaften. Es geht um die Fraven und ihr für die Weiterexistenz der Dörfer unabdingliches Produkt, die Kinder. Also sind Brautpreiszahlungen besonders hoch und incensiv in parrilinearen Gesellschaften. Daß es dabei letzrlich um die Kinder geht, zeigt die Regel, daß der Brautpreis bei der Familie der Frau bleibt, auch nachdem sie ihren Mann verlassen hat, wenn nur ihre Kinder bei seiner Familie bleiben. Bei den Nuer, einem patrilinearen Hircenvolk, ist der Brautpreis hoch. Er beträgt etwa vierzig Rinder. Dieses System der Brautpreiszahlungen ist ein für den Zusammenhalt der Gesellschaft außerordentlich wichriges Instrument, das zum Beispiel auch bedeuter, daß ein junger Mann eine Frau nur im Konsens und Zusammenhalt seiner Familie finden kann. Es dient außerdem als Mittel zum Abbau ökonomischer Unterschiede, denn sobald eine Herde wieder die entsprechende Größe erreicht hat, wird sie für den nächsten Anwärter der Familie als Brautpreis eingeserzt, so daß sich die ökonomischen Unterschiede der Familie reduzieren auf Unterschiede in der Zahl der Frauen, auch von einzelnen Männern, denn regelmäßig fühn dieses System der Braucpreise auch dazu, daß man sich damir mehrere Frauen kauf, wenn man es sich ökonomisch leiscen kann, häufig z. B. bei den Tallensi, weniger häufig bei den Nuer, am wenigsten bei den Lele. Bei den matrilinearen Lele hat sich dafür ein erstaunliches System der sukzessiven Polygynie der alten Männer encwickelt, die ihre Enkelimnen heiraten oder über sie gegen Brautpreiszahlungen in Form von raffia - Palmen-Tüchern verfügen, so daß die jüngeren unter einem Mangel an Frauen leiden müssen, über den sie sich durch die vorläufige Anschaffung einer gemeinsamen Frau für eine Gruppe von mehreren gleichaltrigen Männern hinweghelfen.

Wie auch immer man das Brautpreissystem im Hinblick auf die Siruation der Frau beurteilen mag, jedenfalls verhindert es die Entstehung von ökonomischen Klassenunterschieden, die die Solidaritär der lineage durchbrechen würden. Es gibr allenfalls Unterschiede in der Zahl der Frauen, die die Solidarität der Männer aber nicht entscheidend beeinträchtigt.

Die Verbindung von Mann und Frau in der Familie isc stärker als in Jägergesellschaften. Das hat seinen äußeren Grund im Brautpreissystem, durch das mindestens die Frauen gehalten werden, bei den Männern zu bleiben, bis Kinder geboren sind, weil sonst sie ihre Familie zwingen würden, den Brautpreis zurückzuzahlen. Nach der Geburt der Kinder ist der emocionale Druck zu bleiben, eben der Kinder wegen, ebenfalls ziemlich groß. Dahinter steht jedenfalls der gesellschaftliche Zwang für die Frauen, Kinder zu produzieren, die die ökonomische Weiterexistenz der seß̧aften 
Gruppe garaocieren, dean anders als bei den Jägern ist hier die Regulierung der Größe der Gruppe über Fluktuation sehr viel weniger möglich, der Druck auf die Familie sehr viel stärker. Trotzdem gibe es Trennungen. Meistens verlassen die Frauen die Männer, denn sie sind es, die unterdrückt werden. Die Männer haben das nicht nötig, sie können emocionale Probleme auch häufig durch den Erwerb neuer Frauen lösen.

Selbstverständlich gibr es soziale Normen bei ihnen, gibr es Regeln für die Zugehörigkeit zur lineage, für die Zahlung und Verteilung der Brautpreise, die Exogamie, Sexualtabus, für die Zahlung von Blutgeld bei Törungen oder die Verfolgung von Zauberei. Die meisten Konflikte entstehen aus Streisigkeiten um Frauen oder über die Zahlung von Brautpreisschulden. Es gibr keine Eigentumsdelikte und Vertragsverletzungen, denn sie kennen kaum Privateigentum und jedenfalls keine Verträge in unserem Sinn. Welche Qualizär aber hat ibre Ordnung? Ist sie schon eine rechtliche? Oder sind es Gewohnheiten? Ist es law oder custom? Zunächst: Es gibt keine Gerichte. Es gibt auch keine Zentralinstanz, die die Rechtsprechung in die Hand nehmen könnte. Es gibt nur die Segmente der Gesellschaften, die lineages und Dörfer mit ihren Sprechern und Altescen. Dementsprechend werden auch die Konflikte gelöst, nämlich durch Verhandlungen innerhalb der lineage oder, wenn der Streir darüber hinaus gehi, zwischen den lineages, meisrens durch ihre Sprecher oder Ältesten. Im übrigen gibt es das Prinzip der strukturalen Relativität der Ansprüche. Verlerzungen in sehr großer Nähe der Beteiligten bleiben folgenlos, z. B. Tötungen innerhalb der Familie. Das ist, sagt man bei den Tallensi, wie wenn eine Kuh ihr Kalb zertrampelt. Verlerzungen bei sehr großer räumlicher Entfernung bleiben es in der Regel auch. Je näher die bereiligten lineages zueinander stehen, um so größer ist die Aussicht auf Erfolg von Verhandlungen. Bei großer Entfernung bleibr allenfalls die Selbsthilfe, die aber um so gefährlicher wird, je weiter man sich von seinem Dorf enternt, auch innerhalb desselben Stammes. Es gibr Unterschiede im einzelnen. Bei den Lele ist die Mobjlität der Individuen noch ziemlich groß, fast wie bei Jägern. Die Fluktuation ist also bei ihnen ebenfalls ein häufiger Mechanismus der Konflikelösung. Bei den Nuer gibr es den Leopardenfellpriester als Schlichtungsinstanz für Törungen, die naürlich auch dort nicht als Verbrechen gegen die Gesellschaft angesehen werden, sondern als Verlerzungen der einen lineage durch ein Mitglied der anderen. Bei den Tallensi gibr es auch bei Törungen keine Ausgleichszahlungen, kein Blurgeld, statr dessen die Blucrache zwischen den lineages oder kriegerische Auseinandersetzungen. Andere Streitigkeiten, wenn sie erheblich sind, werden geschlichtet durch Verhandlungen mit den Sprechern der lineages. Das läßr sich allgemein für segmentäre Gesellschaften sagen: Konflikte werden nicht durch gerichtliche Entscheidung gelöst, sondern durch Scblichrung, Selbsthilfe oder durch kriegerische Auseinandersetzung.

Ohne Zweifel steht hinter alldem schon die Vorstellung von Gleichheit und Gegenseitigkeit, die auch bei uns im Recht existiert. Man denke nur an die Brautpreiszahlungen oder die Leistung von. Blurgeld. Allerdings iss es niche eine Gleichheit der Individuen, sondern die der lineages. Es gibt auch die Vorstellung von Ansprüchea der einen gegen die andere, wobei man allerdings beronen muß, daß unsere Vorstellung des Anspruchs ( $\$ 194$ BGB) von Bernhard Windscheid bewußc als nichtscaatliches, rein gesellschaftliches Institur geschaffen worden ist, das unabhängig vom Prozeß gesehen werden soll, unabhängig von der Existenz des Staates, im Gegensatz zum bisherigen akcionenrechtlichen System des römischen Rechrs. Und selbsrverssändlich gibr es in diesen Gesellschaften auch die Vorstellung von ricbrigem und falschem Verhalten, am deutlichsten bei den Nuer mit ihrem Begriff cuong. Jemand bat cuong, wenn er im Recbr ist. Auch Anteile am Brautpreis 
oder bei der Vereilung von Opferfleisch werden als cuong bezeichnet: "Das rechte Hinterbein ist das cuong des Vaterbruders. "Cuong bedeuter "richtig", z. B. das Haus steht richtig, nämlich aufrecht, oder: wein Heró möge aufrecht stehen «, es möge ihm gut gehen. Der Gegensatz ist duer: falsch, Fehler, und zwar der bewußte. Der ungewollte Fehler, die Fahrlässigkeit, ist gwac, besonders bei Tötungen. Das Begriffspaar cuong-duer ist stark verbunden mit religiösen Vorstellungen. Gort ist immer im Recht, cuong, und unterstützt den, der ihm gegenüber und gegenüber anderen Menschen cuong hat, und er straft denjenigen, der bewußre Fehler begeht, duer. Man erinnert sich an Henry Maine und die Entscehung von Recht aus der Religion. Im übrigen gibr es ja auch religiöse Implikationen bej Törungen: Man muß opfern, mit dem Leopardenfellpriester, denn der Gcist des Getöteten ist gefährlich. Es ist allerdings kein Vergehen gegen Gotr oder gegen die Gemeinschaft. Aber was ist nun cuong? Zunächst etwas Objekcives und Subjekcives. Das erinnert stark an unsere Vorstellung von objekcivem und subjeksivem Recht. Aber zunächst muß man darauf hinweisen, daß es als subjektives der Anspruch ist. Aber was ist es als objektives? Recht oder Gewohnheit?

Zur Beantwortung dieser Frage mache ich einen Umweg und beginne mit dem Vercrag. Isr die Vereinbarung über den Brautpreis nicht doch ein Vertrag in unserem Sinn? Nun, zunächst jedentalls nicht $z$ wischen Individuen, sondern zwischen lineages. Aber soweit man von lineage-Eigentum sprechen kann, soweic kann man hier auch von lineage-Vertrag sprechen. Er hat dieselbe Qualitär übrigens wie Vereinbarungen über Blutgeld, die ein Vertrag sind im wahren und ursprünglichen Sinn des Wortes, nämlich im Sinne von sich vertragen, so wie das römische pactum abgeleiter ise von pacisci, pax, der Frieden. Es bedeuter ursprünglich den Friedensvertrag zweier autonomer Gruppen und ist sehrähnlich dem heutigen völkerrechtlichen Vertrag zwischen zwei souveränen Völkern. Ähnlich wie Völker heuce, existieren lineages in segmentären Gesellschaften nebeneinander. Xhnlich können sie Vereinbarungen treffen. Aber ist das Recht? Auch dem Völkerrecht ist seine Qualicät als Recht immer wieder bestritcen worden, weil es keine überstaatliche Zwangsgewalt gibc. Max Weber hat ihm die Rechusqualizät abgesprochen. Auch in unserer heurigen Rechustheorie wird diese Meinung noch vertrecen. Wir können also vorläufig sagen: Die Brautpreisvereinbarung ist ein Vertrag zweier autonomer Gruppen, der keine Rechtsqualität hat. Schon insolern ist sie schlecht zu vergleichen mit dem heutigen Ventag.

Es kommt noch etwas anderes hinzu. Ihre Funkrion ist eine ganz andere. Für einen Mann in segmentären Gesellschaften werden Verträge höchstens zweimal geschlossen, einmal wenn er heiratet, einmal wenn er getötet worden ist. In unserer Gesellschafr, die eine Verragsgesellschaft ist, werden Verträge mehrmals täglich geschlossen, von jedem von uns. Im Jahresdurchschnitr schließr jeder von uns mehr als tausend Verträge ab, das macht im Leben eines jeden von uns mindestens 50000 , meiscens noch sehr viel mehr. Was ist der Unterschied zwischen 2 und 50000 ? Es isc nicht nur die numerische Differenz, sondern eine andere Qualität. In frühen Gesellschatien ergeben sich Produkrion, Konsumprion, Reproduktion und das gesamte tägliche Leben aus dem Status des Menschen in der Gesellschaft, aus seiner Mitgliedschaf in der Horde oder lineage. Bei uns ist es die tocale Freiseczung von allen gemeinschaftlichen Bindungen, die totale Mobilität und das heißt auch Verfügbarkeit, die juristisch konstruiert wird über die Rechtssubjektivicät, das Privateigentum und den Vertrag. Bei uns hat der Vertrag im wesentlichen die Funktion der ökonomischen Organisation der Gesellschaft. In frühen Gesellschaften haben Vereinbarungen über Brautpreis und Blurgeld allein die Funktion einer gleichmäßigen Verceilung heirarsfähiger Frauen und arbeitsfähiger Männer zwischen den Dörfern, 
die im übrigen ohne Verträge produzieren und konsumieren. Insofern hat Henry Maine recht, der in seinem Ancient Law 1861 geschrieben har: "We may say that the movement of the progressive societies has hizherco been a movement from Starus to Contract.r So wie die Braurpreisvereinbarung zu unserem Verrrag, verhält sich auch das cuong der Nuer zu unserem Recht. Es ist die sich selbst regulierende Ordnung früher Gesellschaften, die aufrechterhalten wird durch das Gespräch, durch Selbsthilfe oder freiwillige Schlichoung, norkalls durch individuelle Gewalt. Cuong regelt das selbständige Nebeneinander autonomer Segmence in einer Gesellschaft ohne Zentralinstanz. Es exisciert ohne Herrschaft und wird verwirklicht ohne Herrschaft, ohne Gerichte. Es ist custom, Gewohnheic, nicht Rechr.

\section{Matriarchat und Matrilinearität}

Das Matriarchat ist die Entdeckung Joharn Jakob Bachofens. Ausgehend von einer Bemerkung Herodors über die Lykier, bei denen die Kinder den Namen der Mutrer erhalten und nicht des Vaters, in genialer Interpretation der griechischen Mythen und auf der Grundlage weiterer Nachrichten aus der Anuike nahm er an, die Vorherrschaft der Frauen über die Männer, die Gynaikokratie, sej eine allgemeine Kulturstufe der Menschheir, die bei allen Välkern dem Patriarchat vorangehe, das den endgültigen Sieg darstelle des männlich Geiscigen über das weiblich Scoffliche. Seine Entdeckung schien eindrucksvoll bestärigt, als Morgan einige Jahre später in seiner "Ancient Sociery" die Matrilinearität der Irokesen beschrieb und sie wie Bachofen darauf zurückführe, daß am Anfang der Entwicklung der Familie die Gruppenehe gestanden habe, die Verbindung mehrerex Frauen mit mehreren Männern, so daß immer nur die Abstammung des Kindes von der Mutter sicher sein konnte. Morgan folgte ihm auch in der Annahme, daßs die Namensgebung nach der Mutcer auf eine entsprechend beherrschende gesel lschaftliche Stellung der Frauen schließen lasse, so wie die Namensgebung über den Vater verbunden iš mit der Herrschafr der Männer über die Frauen, folgre ihm allerdings nur zögernd, weil dies eigenclich seiner Grundauffassung widersprach, die Gentilgesellschaft sei frei, gleich und brüderlich gewesen. Schließlich übernahm Engels das ganze in seinem .Ursprunge.

Die Meinung ist heute nicho mehr haltbar. Der Schluß von der Macrilinearieăt auf ein Matriarchar ist falsch. Man kennt heure mehr als hunder matrilineare Gesellschaften. Uberall in der außereuropäischen Welt finden sie sich. In der Regel sind in ihnen die Frauen von den Männern unterdrückt, mindestens scark benacluteiligr. Vielweiberei ist allgemein auch in matrilinearen Gesellschaften verbreitet, "Ämter« werden nur von Männern besetzt. Von einem überwiegenden Einfluß der Frauen kann keine Rede sein, allenfalls in einigen wenigen Fällen, die man nicht verallgemeinern kann. Allerdings sind die Forschungen richt ganz unzweifelhaft, weil meistens nur männliche Ethnologen mic Männern dieser Gesellschaften gesprochen und häufig sich auch für das Frauenproblem nicht interessiert haben.

Wie kommc es zu Marrilinearität und Patrilinearität? Es gibr noch keine sichere Erklärung. Bachofens Lösung, sie mit der jeweiligen Vorherrschaft von Frauen oder Männern gleichzusetzen, ist falsch. Wahrscheinlich hängt die Enrstehung der lineage-Systeme mir der Entstehung der Seßhafrigkeir zusammen. Die Dörfer sind zu klein, um die Geburt ausreichender Nachkommen zu garantieren. Immer wieder trifft man auf das existentielle Interesse, möglichst viele Kinder zu haben, die das Utherleben der Produkcionseinheit und die Versorgung der Alten garantieren. Oft gibr es nicht genug heirassfähige Frauen. Man muß zwischen den Dörfern heiraten. 
Damit entsteht die Frage, welches Dorf die Kinder bekommt. Matrilinearität (: das Dorf der Frau) oder Patrilinearität (: das des Mannes) sind zwei mögliche Lösungen dieses Problems, ohne daß die eine der beiden die ältere sein muß. Damit erhebr sich die Frage nach den Gründen, die zur einen oder anderen Lösung führen. Dazu gibe es amerikanische Untersuchungen, die auch noch nach Matrilokalität und Patrilokalițät unterscheiden. Eines der vielen besonderen Probleme in matrilinearen Gesellschaften ist nämlich die Frage, wo die neue Familie sich niederläßr. Es gibt verschiedene Lösungen. Sie können im Dorf der Frau wohnen, das nenлt man Matrilokalität, oder in einem Dorf der lineage des Mannes, was hier - verkürzt - als Parrilokalizär bezeichnet wird. Kathleen Gough und David Aberle haben auf der Grundlage von damals ${ }_{4}$ sratiscisch erfaßten Gesellschaften festgestellt, daß unter ithen eine besonders große Häufigkeir von solchen mir Garten- oder Hackbau ohne Pflug bescehr, im Gegensatz zu patrilinearen Gesellschaften, in denen die Häufigkeit des Getreidebaus sehr viel höher ist. Die Häufigkeit von Garten- oder Hackbau ist dabei besonders groß in matrilinearen Gesellschaften mit Matrilokalität. Aus diesen Daten leiten sie zwei historische Aussagen ab: Macrilineare Gesellschafren sind entsanden auf der Grundlage einfachen Gartenbaus ohne Pflug. Sie sind bei ihrer Entstehung matrilokal organisiert gewesen. Kathleen Gough fübr beides zurück auf die Arbeitsteilung der Geschlechrer. Beim Gartenbau ohne Pflug isr die Arbeitsleiscung der Frau stärker und wichtiger. Deshalb bleibr sie in ihrem Dorf. Diese Aussagen werden ergänzr von Alice Schlegel. Auch sie arbeiter statistisch, auf der Grundlage von - sehr viel breiter gestreucen - Daten über 66 matrilineare Gesellschaften. Wichtigstes Ergebnis: Die Stellung der Frau ist verhälmismäßig stark, relativ autonom und gleichberechtigt mit dem Mann, teilweise sogar etwas stärker, bei Matrilokalität. Das ist die Regel, von der es Ausnahmen gibr. Die Stellung der Frau ist schwach bei Patrilokalität.

Wenn man versucht, sich das im Wege einer historischen Entwicklung zu erklären, dann liegt es nahe, dabei an die Entscehung des Brautpreissystems zu denken. Deswegen seien die bisherigen Untersuchungen mit einer einfachen Beobachtung ergänzt. Von 43 matrilinearen Gesellschaften mic Marrilokalitär haben nur drei ein Brautpreissystem. Von 71 solcher Gesellschaften mit Patrijokalizät kennen 46 den Braucpreis und 7 erhebliche Arbeitsleisrungen des Mannes an die Familie der Frau, a)so insgesamt 53 Brautpreisleistungen, nur 18 nicht. Daraus läßt sich der Schluß zjehen: Mir dern Ubergang zur Patrilokalität entsceht der Brautpreis, der die Frau zum Objekt macht und ihre Situacion entscheidend verschlechtert. Wo die Gründe für diesen Ưbergang liegen, das muß zunächst noch offen bleiben.

Ein Matriarchat hat es also nie gegeben, auch matristische Gesellschaften nicht. Die Stellung der Frau war am besten in Jägergesellschaften und in matrilokalen matrilinearen Gesellschaften. Sic har sich dann zunehmend verschlechtert, besonders in solchen mit Getreidebau. Matrilinearität und Patrilinearicät sind Regelungen für die Verteilung der Kinder in seßhaften Hauswirtschaften. Diese Verteilung richrete sich zunächst nach dem Ort des Hausstandes der neuen Familie. Dort, wo sie wohnten, dort bleiben auch die Kinder. Der Ort des Hausstandes ergab sich zunächst regelmäßig aus der An der Landwirtschaft und der Gewichrung des jeweiligen Arbeitsanteils der Geschlechter. Im günstigsten Fall, nämlich bei matrilokaler Marrilinearitä, konnte dies aber nur eine starke Gleichstellung der Frau mit dem Mann bedeuten, nicht ihr gesellschafrliches Übergewicht. Eine entscheidende Verschlechterung ihrer Situation bedeutece die Entstehung des Brautpreissyscems. Ursprünglich nur in patrilinearen Gesellschaften üblich, enestand es auch in macrilinearen beim Ubergang von der ursprünglichen Macrilokaliät zur Patrilokalizät. Durch diese Art der Zirkulation erhateen Brautpreis und Frauen einen eigenen 
Sachwert, der sich schnell verselbständigt und durch Verfälschung der Zirkulation gehäuft werden kann, was regelmäßig nicht zur Häufung ökonomischer Macht führr, sondern zur Häufung von Frauen im Besitz eines Mannes, die dann aber ebenfalls Macht bedeuteq, die früheste Entstehung von quasi-inscitutionalisierter Macht in der menschlichen Geschichte. Die ökonomischen Gefahren konnten von den egalizären segmentären Gesellschaften noch weitgehend gemeistert werden, durch Zerstörung oder Neutralisierung von Heirarsgut, sofern es nicht sowieso gleich wieder in die Zirkulation gegeben wurde, und durch Redistribution in anderer Weise, durch freiwillige Verteilung oder Grabbeigaben. Die sexistischen Gefahren haben auch sie nichr gemeister. So entstand Macht in der überlegenen Stellung des Mannes gegenüber der deklassierten Frau oder gegenüber einer erst recht deklassierten Vielzahl von Frauen und in der sozialen Kontrolle der Alten über diese Zirkulation der Reproduzentinnen.

\section{Die Entstehung von Herrschaft und die Anfänge des Staates}

Trozzdem. Auch segmentäre Gesellschaften sind, mindescens unter Männern, bewußt egalitär. Sie leben, wie Morgan es beschrieben und Clastres formuliert hat, als "Staatsfeinde $\alpha$, mit vielen psychischen und materiellen Instrumentarien zur Verhinderung der Entstehung von organisierter Machr, von der Lächerlichkeit bis zur Redistriburion. Sie leben freiheiclich, egalizär, anarchisch. Wie aber entstehen nun aus segmentären Gesellschaften kephale, aus egalieären Gesellschaften unegalitäre? Wie entsteht organisierte Macht, Herrschaft? Das ist eines der spannendsten Kapitel in der Geschichce der Menschen, und es hat auch schon viele Anrworten gegeben auf diese Frage. Generationen von Philosophen, Historikern und Anthropologen haben sich mit ihr beschäftigt. Die vielen Antworten, die sie gegeben haben, bedeuten wohl auch heute noch einen Teil der Lösung dieses Problems. Es gibt eben viele Möglichkeiten, keine einheitliche Antwort. Die erste stammt von Heraklit, die erstaunlichsce von Ibn Chaldun. Für Heraklit war der Krieg der Vater jeder Entwicklung. Ibn Chaldun aus Tunis hat in der zweiten Hälffe des i 4 . Jahrhunderts eine Einführung in die Geschichre geschrieben und vor sechshunder Jahren schon formuliert, was heute als eine der wenigen gesicherten Erkennenisse der Ethnologie über die Entstehung von Herrschaft angesehen werden kann, daß nämlich der Staat entstehe aus der Unterwerfung f́iedlicher Ackerbauern durch kriegerische Hirtenvölker. Rousseau nahm an, es sei ein Verrag gewesen zwischen friedlichen Menschen. Für Hobbes var der Staat die einzige Möglichkeir, von Anfang an den Krieg aller gegen alle zu verhindern, für Hegel das Ergebnis der Enrwicklung ejner objekriven Idee, und Morgan und Engels baben ihn, äbnlich wie Hobbes, erklärt aus der Notwendigkeit eines schürzenden Daches für die Gesamrgesellschaft, deren einzelne Segmente durch das entsrehende Privareigenrum der Männer zerschlagen wurden und deshalb für die einzelnen den Schutz nicht mehr geben konnten, den sie bis dahin gegeben hatten. So weit zur Geschichre dieses Problems.

Die Morgan-Engelsche Theorie zur Entstehung des Staates enthält eine allgemeine Beobachtung, die auch heute noch richtig ist. Morgan und Engels nahmen an, die Entstehung des Privateigentums - der Männer am Vieh - hätte die lineage-Struktur zerstört und zur Entscehung des Staates, von Kephalizär, geführt. Das ist falsch, denn wir kennen viele kephale Gesellschafien ohne Privaceigentum. Aber die Beschreibung des äußeren Vorgangs stimmt; es ist immer dasselbe: Kephalität encsrehc durch Zerstörung der Segmente. Auf der unteren Ebene wird eine Vielzahl von Individuen freigesetzt und es entsteht über ihnen als einheicliches Dach eine 
herrschaftliche Zentralinstanz, deren Ziel es immer sein muß, die Segmente völlig zu beseitigen und die Individuen zu individualisieren, zu atomisieren. Die Segmente werden zunächst zu mittleren Instanzen, die allmählich beseitigc werden. Was bleibr, ist der Staat und das Individuum. Das ist das äußere Bild, das immer das gleiche ist, in der Geschichte und in der Entwicklung echnologisch früher kephaler Gesellschaften, soweit man sie zurückverfolgen kann. Aber gerade hier ergibr sich und ebenso aus der Geschichte der Palast- und Tempelwirtschaft Agyprens, Mesopotamiens und der minoischen und mykenischen Gesellschafh daß das Privateigentum durchaus nicht der Motor dieser Entwicklung gewesen ist. Hier hat es entweder gar kein Privareigentum gegeben oder in nur unerheblichem Umfang, der jedenfalls die gesellschaftliche Entwicklung nicht entscheidend beeinflußte. Deshalb hat Karl August Wittogel für diese Palast- und Tempelwirtschaften seine shydraulische Theorie « aufgestell, nach der es die Norwendigkeir eines zentralen Bewässerungssystems gewesen sei, die den Staat hervorgebracht habe, weil er allein die Lenkungsund Leitungsaufgaben erfüllen konnte. So einleuchtend sie auf den ersten Blick zu sein scheint, sie erklärt letzclich gar nichts. Sicherlich sind es kephale Instanzen gewesen, die in vielen dieser Gesellschaften die zentrale Bewässerung organisien haben. Aber sie müssen nicht zu diesem Zweck geschaffen worden sein, sondern werden das in Angriff genommen haben, nachdem sie, aus Gründen, die wir erst noch angeben müssen, als kephale Instanzen entstanden waren. Ohne Erkärungswert ist es auch, wenn Gordon Childe, ebentalls für diese Gesellschaften in Mesopotamien und Ägypten, die "urbare Revolution $\alpha$ beschreibs die die neolithische ablöst. Wesentliches Moment des Urbanismus sei die Entwicklung einer intensiven Nahrungsmittelproduktion gewesen, die nicht nur den Bedarf einer dichten Bevölkerung deckre, sondern auch noch einen Ubberschuß ergab, ein nkonzentriertes soziales Surplusa, zur Aufrechrerhaltung der Herrschaft einer EliteHierarchie und eines repressiven Staates. Auch das erklärt allenfalls die Möglichkeit der Existenz von Herrschaft, nicht die Ursachen ihrer Entstehung. Verlassen wir also die Archäologie und wenden uns den Erhnologen zu.

Ihre sicherste Theorie ist die der Erobenng, die Theorie Ibn Chalduns. Kriegerische Hirtenvölker unterwerfen friedliche Ackerbauem. Es gibt viele Beispiele, die mejsten in Ostafrika. Immer sind es hamitische Viehzüchrer gewesen, die Bantu-Ackerbauern unterworfen haben. Das läßt sich rekonstruieren aus der Schichtung dieser Gesellschaften, ihrer ethnographischen Sprachverteilung und aus ihren Legenden. Ein gures Beispiel ist das Königreich Ankole in Uganda. Die Bahima überfallen die Bairu, uncerwerfen sie, bleiben in deren Gebiet und leben zwar weiter von ihrem eigenen Vieh, erhalten aber auch Tributleistungen, Hirse, Bier und Arbeitskraft. Das verändert die ursprünglich egalitäre Ordnung der Bahima. Die ständige Unterwertung der Bairu fordert von ihnen verstärkte Zusammenarbeit und die Einrichtung einer obersten Führungsinstanz. Es entwickelt sich eine hierarchische Ordnung mir einem König an der Spirze, der zu seinem Schutz eine Truppe von mehreren hundert speziell ausgebildeten Kriegern an seiner Seite und gemeinsam mit den Häupdingen die Rechisprechung übernommen hat. Ahnlich, wenn auch sehr viel komplizierter, ist es bej den Nupe in Westafrika gewesen. Von den erobernden Fulani war der eine Teil, die Hirten, sehr friedlich, der andere, die Ackerbauern, bestand aus fanatischen Moslems mic starker Tendenz zu aggressiven heiligen Kriegen. Außerdem gab es hier im wesclichen Sudan schon starke europäische Einflüsse. Man handelte mic Gold und Sklaven und hatte europäische Waffen. Deswegen ist die Eroberungstheorie allein in diesem Fall nicht ausreichend, um die Entstehung dieses Schwarzen Byzanz, wie Nadel es genannt hat, zu erklären. Aber die Erobenungstheorie hat auch allgemeine Schwächen, selbst im Fall des viel 
günstigeren Beispiels von Ankole. Der Krieg allein muß nicht unbedingt die Ursache der Entstehung von Herrschaft sein. Wohl aber kann eine entstehende Herrschaftsinstanz den Krieg provozieren und damic ihren milizärischen Status verbessern. Daß Krieg in funkrionierenden segmentären Gesellschaften durchaus nicht die Entsrehung von Herrschaft zur Folge haben muß, zeigen die Nuer mix ihren ständigen Eroberungszügen gegen die Dinka. Thre egalitäre Strukrur blieb davon unberühre.

Man unterscheidet im übrigen zwischen exogenen und endogenen Ursachen. Für die Bairu ist der Eroberungskrieg der Bahima ohne Zweifel eine exogene Ursache gewesen, die die Herrschaft von außen in ihre Gesellschast hineingerragen hat, für die Bahima eher eine endogene, die in inneren Bedingungen ihrer eigenen Gesellschaft zu suchen ist. Solange man nicht konkrete Gründe benennen kann, hat die Unterscheidung ohnehin wenig Sinn. Das zeigt das Beispiel der Aschanti, das häufig für die endogene Theorie genannt wird. Sie waren, als sie am Ende des 19. Jahrhunderts von den Engländern unterworfen wurden, eine milizärische Konföderation von etwa zwanzig halbautonomen Stämmen, die unter einem gemeinsamen $\bowtie$ Königu, dem Asante Hene in Kumasi, an der Goldküste lebten. Der Asante Hene hatte nicht nur den militärischen Oberbefehd, sondern auch zivile Herrschafusfunktionen, z. B. die Rechrsprechung für Kapitaldelikte an sich gezogen. Die Konföderation unter Führung des Stammes von Kumasi war zustandegekommen im 18. Jahrhunder, im Kampf gegen Nachbarscämme, von denen sie angegriffen und mit Triburforderungen bedrohe wurden. Anders als in den Königreichen Ankole oder Nupe gibc es bei ihnen keine soziale Schichcung von Eroberern und Eroberten. Ihre segmentäre Struknur ist noch ziemlich stark, steht erwa im Gleichgewicht zur kephalen. Sicher kann man ausschließen, daß die Entscehung der Herrschaft bei ihnen endogene ökonomische Ursachen gehabt hat, gerichtet war auf ökonomische Vorreile oder Ausbeurung. Es war keine ökonomiscbe, sondern wie man heure zu sagen pflegt, eine "politische" Herrschafr.

Diese "politische" Theorie der Entsrehung von Herrschaft wird heuce in der Ethnologie weitgehend vertreten, von Sigrist und Clastres erwa, zuletzt von Service. Sie wird weitgehend richtig sein, soweit man ihre Aussage in dem Sinn versteht, daß in all diesen Fällien »ökonornische" Ursachen auszuschließen sind. Im übrigen ist sie aber ähnlich nichrssagend wie die endogene Theorie vom sdemographischen Druck «. Diese Theorie stürzt sich auf die Beobachtung, daßs segmentäre Gesellschaften meistens kleiner sind als kephale. Mit dem Wachsrum der Bevölkerung entsrehe die Norwendigkeit von Steuerungsmechanismen, gleichzeicig entscehe dann die Möglichkeit des Abschöpfung von Surplus. Auch diese Theorie ist letztlich nur eine Beschreibung, keine Erklärung. Zunächst zeigt ein Blick auf die stark herrschaftlich organisierte Gesellschaft der 12000 Trobriander im Vergleich mit den 300000 Nuer und ihrer völlig intakten egalitären Ordnung, daß sie auch nur einen beschränkten Beschreibungswert hat. Und im übrigen muß man einwenden, daß Kephalïät mit ihren Steuerungsmöglichkeiten die ökonomische Struktur stark verbessern und eine verbesserte Okonomie auch zu demographischem Wachstum führen, daß, mit anderen Worten, das Verhältnis von Ursache und Wirkung gerade umgekehrt sein kasn. Wie man wohl allgemein sagen muB, daß Veränderungen der ökonomischen Verceilung nie die Ursache, sondern nur häufig die Folge der Entstehung von Herrschafr sind. Man kann eine begrenzre Zahl von Erscheinungen benennen, die oft oder regelmäßig mit der Entstehung von Herrschaft verbunden sind, endogene wie demographischer Druck und exogene wie Krieg, aber wir sehen noch nicht hinter diese Erscheinungen. Sicherlich gibr es nicht nur eine Ursache. Auch diejenige, die besonders in der klassischen marxistischen Literatur immer 
wieder benannt worden ist, wird es wohl nicht sein. Die ökonomische Theorie läßt sich nirgendwo bestätigen. Für die Echnologen scheint die Angelegenheit damit erledige zu sein, daß sie die aufkommende Herrschaft als politische bezeichnen, womit man leczlich auf anthropologische, in der Nacur des Menschen liegende Gründe zurückgreift, was wenig plausibel ist, weil gerade für Jägergesellschaften und segmentäre Gesellschaften das Gegenteil festgestellt worden ist. Man muß also weitersuchen. Eine mögliche Ursache ist, soweit zu sehen, noch von niemandem in Betracht gezogen. Die sexistische. Besonders die Vertreter der social anthropology sind durch die segmenrären Geselischafren gegangen und haben das hohe Lied ihrer Egalität gesungen, sicherlich auch zu Recht, soweiz sie die Situation der Männer beschrieben haben. Die Freiheir der Männer ist bei ihnen in der Tat sehr groß. Aber ebenso groß ist oft die Unfreiheir der Frauen. Bei ihnen hörr die Egalirät schnell auf. Man denke nur an die Frauen der Tallensi, an die Mache der alten Männer über die jungen Frauen bei den Lele. Mit zunehmender Unrerdrückung der Frau beobachten wir ein zunehmend aggressives Verhalten der Männer zueinander. Die Gewalt nimmt zu. Wenn es auch kein ökonomisches Ziel für das Streben nach Machtpositionen gibt, weil die egalitären Mechanismen insoweit funktionieren, so gibt es doch ein sexistisches, die Häufung des Besitzes an Frauen. Außerdem haben Macht und Herrschaft infizierende Eigenschaften. Wenn es in einer Gesellschaft erst einmal institucionalisierte Macht gibr, breicet sie sich leicht in andere Bereiche aus. Und diese Herrschaft gibr es eben auch in den egalitären segmentären Gesellschaften in der Form der institutionalisierten Macht des Mannes über die Frau. Warum solle sie sich von dort nicht auch in den Bereich der Männer ausdehnen, in Macht von Männern über Männer. Konkurrenten sind sie insoweir immer gewesen. Als ökonomische Konkurrenten haben sie sich in frühen Gesellschaften nie verstanden. Herrschaft ist sicher nicht dadurch entstanden, daß eine Zirkulation von ökonomischen Gütern vezfälscht wurde. Aber wahrscheinlich ist sie in höherem Maße, als wir es heute ahnen, ausgebreiter worden durch die Verfälschung der in segmentären Gesellschaften von Meillassoux beschriebenen Zirkulation der Frauen.

\section{Das Recht, seine Entstebung, Funktion und weitere Entwicklung}

Wer die Herrschaft har, wird ein anderer. Der König von Ankole mit seiner Leibgarde von einigen hundert Mann hat eine andere Stellung, eine andere Funkrion als der Alteste eines Lele-Dorfes, der na'am der Tallensi oder der sbull einer Nuer-Siedlung. Wenn er mit seinen Häupclingen zu Gericht sitzı, sieht es nur äußerbich ähnlich aus wie bei einer Beratung in einem Dorf der Lele, Tallensi oder Nuer. Sein Urteilsspruch ist etwas anderes, nicht vergleichbar mir dem Versuch einer Schlichtung durch den Leopardenfellpriester, auch wenn es um die gleichen Konflikte geht. Nirgendwo wird das so gut deutlich wie bei den Aschanti. An ihrem Beispiel kann man verstehen, wie frühestes Recht entsteht und sich ausbreiter, und man kann daneben noch die alve Ordnung der Gewohnheiten sehen und wie sie langsam von ihm zurückgedrängt wird.

Ihr Gebiet ist heute eine der acht Regionen der Republik Ghana. Sie betrieben im wesentlichen Ackerbau. Es gab viel Gold, das ihre weitere Entwicklung stark bestimmte, und viel Krieg, zum großen Teil zur Sicherung ihres Fernhandels, der monopoliscisch in der Hand ihrer Häuptinge lag. Nach Norden exportierten sie die Kola-Nuß und von dort importierten sie Stelaven. Ưber die Küste wurden Sklaven und Gold exportiert und europäische Waren importiert. Trotz des starken Zentralismus mir dem mächrigen König von Kumasi und seinem Goldenen Stuhl ais 
Symboi der Einheit aller Aschantistämme an der Spitze, waren sie noch korporativ von unten nach oben aufgebaut, letzelich eine Hauswirtschaft ohne Schrift und Geld. Die autonomen dezentralen Elemente waren stark geblieben, die einzelnen Stämme und die matrilinearen lineages. Die lineage, abusua, ist bei ihnen ziemlich groß, rechnet ihre Abstarmmung über sechs bis achr Generationen zurück. Das Amr ihres Altesten ist, wie alle "Stühle« der Aschanti bis hinauf zu dem des Asance Hene, reils erblich und reils auf Wahl gegründer. Erblich isc es insofern, als Voraussetzung für die Wahl die Zugehörigkeit zu einer engeren Gruppe innerhalb der größeren ist, deren Amc beseczt und von der gewähit werden soll. Die abusua als lineage zerfält wieder in kleinere Familieneinheiten, Dorfgemeinschaften, die über drei bis vier Generacionen zurückrechnen und fie (Haus) genannt werden. Das fie ist die eigentliche ökonomische Einheir, der auch das Land zugerechnet wird, das sie bebaut. Die lineage, ihr Sprecher und sein Rat und die zu ihr gehörenden Hausgruppen sind die Grundlagen der Gesellschaft der Aschanci. Hier wird das Eigentum am Land weitergegeben und über Streitigkeiten entschieden. Hier hat die alie Ordnung des efiesem ihren Platz und wird die Einigung mit anderen lineages herbeigeführt, wenn Konflikte nach außen entstehen. Auch Heiraten werden erst wirksam, wenn der abusua panin der Frau mit dem des Mannes sich geeinigt und festgestellt hat, daß Exogamievorschriften durch die beabsichrigte Verbindung nicht verletzt werden. $\mathrm{D}_{2}$ die Gesellschaft von unten nach oben aufgebaut ist, können die Häuptlinge auch abgewählt werden, was häufig geschiehr. Und es gibr das Prinzip der Autonomie der jeweils unceren Einheit. Der Asante Hene kann niche selbst in die Stämme hineinregieren, sondern nur über ihre Häuptlinge, die in seinem Rat sitzen, als seine Verbindungsleute zu diesen Einheiten der Union. Der Häuptling kann nichr direke in die Untergruppe des Stammes einwirken, sondern nur über den birempong, der die Dorfgruppen vertrit, oder den abusua panin, den Sprecher der lineage, die beide in seinem Rat mitwirken und die Verbindung herstellen von unten nach oben und umgekehrt. Rattray hatte noch gemeint, in dieser pyramidenförmigen Gesellschaft die feudale Ordnung des alten England wiedererkennen zu können. Aber dort beruhte der Feudalismus ja auf dem Obereigentum des Königs am ganzen Land, mit dem seine Herrschaft verbunden war. Die Gesellschaft war von oben nach unten organisiert. Die Aschanti bieren nur äußerlich das gleiche Bild. Ihre Ordnung gründer sich auf den Landbesitz der unteren Gruppen, der lineages, später des fie. Sie ist von unten nach oben aufgebaut, wie man aus den Wahlvorgängen leicht erkennen kann.

Aus dem Gleichgewicht von Kephalizät und segmentärer Ordnung ergibt sich ein seltenes, gleichwertiges Nebeneinander von Recht und Gewohnheit. Die Aschanti uncerscheiden zwischen einem kleinen Bereich von Verlerzungen, über die zencralistisch entschieden werden muß, vom Häuptling, dem birempong oder zunehmend vom Asante Hene, und einer großen Zahl anderer Konflikte, die innerhalb der lineage beigelegt werden können. Der erste ist der Bereich des oman akyiwadie, das bedeutet sinngemäß etwa nwas der Stamm (oman) nicht ertragen kann, mißbilligt $\alpha$. Der zweite Bereich wird efiesem genannt, von fie, das Haus, und kann ungefähr mit "Hausangelegenheiten « übersetzt werden.

Oman akyiwadie ist der Bereich, den wir öffendliches Stralrecht nennen würden. Es sind Verleczungen der Ordnung, die fïr die Gemeinschaft als so gefährlich angesehen werden, daß sie mit schweren Sankrionen eingreilen muß, um den Zorn der Götrer zu besänfrigen. Auch die Görter denken zentralistisch, wenn es Herrschaft gibr. Es geht in erster Linie um Törungen. Hier haben die lineages ihre Komperenzen nach oben abgeben müssen. Das ist ein wichtiges Zeichen für die politische Strukcur einer frühen Gesellschaft, meistens der Beginn der Zerschlagung der 
Gentilstruktur durch die Zencralinstanz. Mit der Einführung der Popularanklage durch jedermann hat Solon im 6. Jahrhundert in Achen die alten Geschlechter entscheidend getrotten und ihre endgülrige verfassungsrechrliche Entmachung eingeleitet. Die Asante Hene der Aschanti waren ohne Zweifel auf dem Wege dorthin. Auch andere Verlezzungen der Ordnung wurden schon von ihnen zentraliscisch verfolgt, etwa der Inzest und andere Tabuverletzungen. Regelmäßig wird die Todesstrafe verhängt. Einige Vergehen werden wnur* mit schweren körperlichen Verstümmelungen geahndec.

Mit dem Wachsen der herrschaftlichen Struktur ihrer Gesellschaft nahm auch die Härte ihrer Strafen zu. Zunächst waren die Häuptlinge, später nur der Asante Hene zuständig. Die Rechtsprechung hatte sich nämlich im Laufe der Zeit zu einem Privileg entwickelt, das für den „Goldenen Stuhla von großer Bedeutung wurde und die Ausbreitung von Recht stark beeinflußte. Es war üblich geworden, daß die zum Tode Verurteilten oder ihre lineage sihren Kopt vom König zunückkaułen « konnten. Der Asante Hene konnte nur im Kriegsfall von den Stämmen Abgaben verlangen. Diese Einnahmen aus der Verhängung von Todesstrafen wurden eine seiner wichtigsten Finanzquellen. Die Monarchie, die zunächst im wesentichen Außentunkcion hatte, verwandelte sich langsam zur selbständigen Macht nach inner. Und das Recht diente dabei zunehmend auch der Finanzierung eines königlichen Apparacs, der immer mehr Prozesse in Gang seczte und darauf drängte, daß die einnahmerrächrige Todesstrafe verhängt wurde. Die Prozesse fanden stat vor dem Rat des Asante Hene in Kumasi, mit einem der Sprecher des Rates, okeyarne, als Ankläger. Es gab gewisse Regeln für den Ablauf des Verfahrens. Die Vollstreckung lag in den Händen eines speziell zu diesem Zweck beim »Goldenen Stuhla beschäfrigten Personals. Es war also voll ausgebildetes Recht in den Händen einer Zentralinstanz, die dafür auch mit dem Monopol der physischen Gewalt ausgestatter gewesen ist. Aber dieses Recht hatre sich noch niche über die ganze Gesellschaft ausgedehnt. Es blieb beschränkt auf den Kernbereich schwerer Delikte und war zunächst auch nur strafrechulicher Nanr. Und es existierce daneben die alte Ordnung der segmentären Gesellschaft.

Im Gegensatz zur zentralen Gerichtsbarkeit des oman akyiwadie ist die Beilegung von Srreitigkeiten des efiesem eine Angelegenheit der lineage. Es wird immer, und das ist der Unterschied zum blutrünstigen oman akyiwadie, eine einverständliche, friedliche Lösung gefunden, ohne physische Sankcionen. Weder der abusua panin noch der fie panin haben je das ius virae necisque gehabe wie die Häuptlinge oder der Asante Hene. Es geht im efiesem um eher private Verletzungen, Privardelikte. Es geht um Streicigkeiten wegen Frauen, Täclichkeiten, Beleidigungen. Diebstahl spielte nur eine Rolle, wenn er außerhalb der lineage begangen wurde. Waren es geringere Streitigkeiten, wurde irgendeiner der Äteren zum Schlichten bestelit. In schwierigeren Fälen entschied der abusua panin, möglicherweise auch mit dem ganzen Rat der lineages. Es gab keine fescen Regeln. Die Einigung aurde einverscändlich erzielt. Manchmal wurden Bußen vereinbart, als mpaca, Besänfrigung. Auch hier gab es keine festen Sätze. Ein Huhn oder ein paar Eier waren schon geeignet, "die Seele des Verletzten zu waschen. * Das höchste war der Ausgleich mit ein wenig Goldstaub.

Rattray hatce dieses efiesem noch gemeinsam mit dem oman akyiwadie unter den Begriff des "Law and Procedure" gebrachı, obwohl er die Uncerschiede im einzelnen ziemlich $\mathrm{klar}$ herausstellte. Busia hat dann den grundsätzlichen Unterschied der beiden Bereiche noch stärker beront und die selbstregulierende Funkcion des efiesem deutlich beschrieben, indem er daraú hinwies, daß es freiwillig akzepciert wurde und ohne rechtliche Sankrionen existiert. Aber erst Hoebel ist es gewesen, 
der erklärte, daß es sich beim oman akyiwadie um law, beim efiesem aber um custom handle.

Wir haben bisher nur den delikcischen Bereich behandek, sowohl im oman akyiwadie als aucb im efiesem. Dieser delikrische Bereich enchält, noch ungetrennt, das Strafrecht und das private Schadensersatzrecht. Es ist "Privarstrafe" (Ernst Levy), die sich, überall in der Geschichte des Rechts, erst später in Strafe und Schadensersatz trennt. Wo bleibr aber der eigentliche Bereich unseres Privatrechrs? Wo bleiben Streicigkeiten um Eigentum, Erbschaften, Verträge? Schon Raturay hatte beobachtet, daß sie völlig fehlen. Es gäbe kein Privatrecht in unserem Sinn, keine Grïnde für Streicigkeiten dieser Art. Er hatte im wesentlichen Rechr. Das Eigentum war innerhalb der lineage gebunden, auch am Land. Die Vererbung wurde innerhalb der lineage geregelt, obne daß es zu ernsthaften Streitigkciten darüber kommen konnte, wenn der Rat der lineage seine einverständliche Entscheidung getroffen hatte. Spärer, als es in gewissem Umfang Privareigentum neben dem lineage-Eigentum gab, entstand tarsächlich die Frage der Vererbung auf die eigenen Kinder, die in matribnearen Gesellschaften immer problemarisch ist. Es gab dann die Möglichkeit eines unverbindlichen Testaments zugunsten der eigenen Söhne, das von den wirklichen matrilinearen Erben der lineages des Varers häufig anerkannt wurde, zugunsten seiner Kinder, die zur lineage ihrer Mutter gehörten. Wenn sie das nicht taten, dann tracen die Sprecher der beiden lineages zusammen und verhandeiten über eine Lösung des Konflikts, der dann in der Regel zugunsten der matrilinearen Erbfolge gegen die Kinder entschieden wurde. Aus Eigentum und über Erbschaften entstand also kein wjuristischer* Streit. Sie blieben im Rahmen der segmentären Ordnung. Und es gab im Rahmen der Hauswirtschaft auch grundsäızlich keine Verräge. Die vichrigste Ausnahme ist das Darlehen.

Es gab viel Gold bei den Aschanti. Es war zwar im wesentlichen wichtig für den Fernhandel, aber es war auch schon in die eigene Gesellschaft gedrungen, ohne daß man von Geldwirtschaft sprechen kann. Es war eine Hauswirtschaft, die außerhalb des üglichen Lebens und Bedarts schon Elemente des Tausches aufgenommen hattc. Der Fernhandel war teilweise in die eigene Wirschaft einbezogen, als Bartausch, ohne Distanzwirkungen in der Zeit. Als einziger Vertrag mit zeitlicher Distanz existierze bei ihnen das Darlehen. Es wurden auch Pfänder dafür gegeben und Bürgen gescelir. Wurde eine Schuld nichr bezahlt, dann scheint es üblich gewesen zu sein, daß die lineage den Schuldner zur Disposition der lineage des Gläubigers stellte, ihn in die Schuldknechtschaft gab, mit der Einschränkung allerdings, daß er als Aschanti nicht in ihrem Gebiet als Sklave leben durfte, sondern nach außerhalb verkauft werden mußte. Die Parallelen mit dem frühen Rom liegen auf der Hand. Auch die Regelung von Streicigkeiten über derartige Darlehen lag in der Hand der lineages, wurde einvernehmlich getroffen. Dabei gab es eine gewisse Parallele zur früheren Regelung von Konflikcen wegen Tötungen. Auch dort war früher die Lösung, daß der Verletzer regelmäßig der lineage des Geröreten ausgeliefert und von ihr in die Sklaverei verkauft wurde. Es gab keine direkten Blurgeldzahlungen. Und hier wie dort gab es die Tendenz zur Zentralisierung, zur Verrechulichung. Es gab, wie beim Asante Hene, auch eine gerichtliche Funktion der Stammeshäuptlinge und ihres Rates. Die wichcigsten Fälle dieser Gerichtsbarkeit waren Beleidigungen oder Verfluchungen des Häuptlings, sie standen unter Todesscrafe, waren oman akyiwadie. Hier setzre man ein, um Streitigkeiten über Darlehen aus dem efiesem herauszubringen, justiziabel zu machen. Beide Parteien sprachen Verfluchungen des Häuptlings aus, die sie unter die Bedingung scellten, daß sie in diesem Screic um das Darlehen im Unrecht seien, sinngemäB: Wenn ich hier im Unrecht bin, dann ist auch der Häuptling ein Lump. Auf diese Weise zwang man das Stammesgericht, bei 
der Verhandlung über die Verfluchung auch die Frage ihrer negativen Bedingung zu untersuchen, also zu klären, wer von beiden im Streit um das Darlehen im Recht gewesen war. Der nämlich mußte freigesprochen, der andere verurteilt werden. Eine Verurteilung zur Leistung gab es auf diese Weise nicht, und daß am Anfang der Entwicklung zur Verrechtlichung von Darlehensforderungen bei den Aschanti die Todesscrafe stand, braucht nicht zu verwundern: auch im frühen Rom konnte der Gläubiger seinen Schuldner tören, wenn nicht gezahlt wurde. Die Verrechrlichung des Darlehens, das wohl meistens in der Encwicklung des Privacrechis am Anfang steht, wird geleister über das öffentliche Strafrecht des oman akyiwadie, das die Verletzung von Vertragspflichten behandelt wie andere deliktische Verlezzungen. Auf diese Weise entstand ein Keim von Privatrecht, entstand auch bei den Aschanti der erste Anfang des Vertrages aus dem Delikt.

Auch am Beispiel dieser Darlehensstreivigkeicen wird die allgemeine Funktion von Reche in seiner Eststehung deutlich. Es bedeutet die Entmachtung der Gentiverbände, die Durchsetzung von zenuraler Herrschaft gegen mitrlere Gruppen, die allmählich aufgelöst werden. Recht hat die Funktion der Freisetzung der einzelnen, die nun zu allen anderen in der gesamten Gesellschaft in ein rechrliches Verhältnis treten, das direkt von der Zentralinstanz vermittelt wird. Diese Freisetzung ist ein hiscorischer Prozeß, den Henry Maine als die Entwicklung vom Status zum Vertuag beschrieben hat und der bei den Aschanti sehr deutlich erst noch an seinem Anfang stand. Am Anfang der Entwicklung des Rechts steht das Delikc, das in einer ungetrennten Verbindung von privater Verletzung und Auflehnung gegen die öffentliche Ordnung gesehen wird. In seiner Verbindung mir der Herrschaft bedeuter die Entstehung von Recht gegenüber der aiten Ordnung der Gewohnheiten die Veränderung zu einer neuen Qualität der Beziehungen der Menschen zueinander, die Vereinzelung, die Auflösung von Solidarität. Die Entstehung von Rechr ist aber nicht nur eine qualizarive Veränderung, sie ist auch der Beginn eines quancitativen Prozesses, der als scändige Ausbreitung von Recht die Verstärkung und Ausweitung von Herrschaft begleirer. Immer mehr Bereiche, die bisher nicht vom Recht erfaßt waren, werden verrechclicht. Bei den Aschanti ist dieser Prozeß im Keim deurlich zu erkennen an der Verrechrlichung des Darlehens. Aus dem Delikı encsteht der Vertrag. Der Vertrag weitec sich aus. Es entstehen neue Verträge, die immer stärker das tägliche Leben bestimmen. Am Ende dieses Prozesses steht die Verrechulichung der gesamten Gesellschaft, die totale Entsolidarisierung. Ihn zu beschreiben iš nicht Ziel dieses Berichts, der nur die ersten Anfänge aufzeigen wollce. Und so bleibc vorerst als Brücke zur Gegenwart die Vermunung, daß Dezentralisierung und Abbau von Herrschaft nicht unbedingt negativ sein müssen und Staatsfeindschaft auch in Zukunft erwas Positives sein kann, die Bildung kleinerer Einheiten in industrialisierten Gesellschaften zwar vielleicht nicht der Weisheit letzter Schluß, als Modell einer humanen Gesellschafr aber sicher auch nicht auszuschließen sein wird und daß jedenfalls die Entwicklung zu immer größeren Einheiten zu stärkerer Verrechtlichung und nichc zu mehr Menschlichkeit führen muß.

\section{Literaturhinweise}

Dic vier grundlegenden Werke des 19. Jahrhunderts: Lewis Henry Morgan, Ancient Sociery, gibe es als Nachdruck der 1908 erschienenen Überselzung von Kauzsky und Eichhoff, Dic Urgesellschaft, Veriag Andreas Achenbach Lollar, 1976; Henry Sumner Maine, Ancient Law, I\$6I, isc nicht überserzh man benutzt einc der vielen späteren englischen Ausgaben; Johann 
Jakob Bachoten, Das Murterrecht, 3. Aufl, 1948; Friedrich Engels, Der Urspnung der Familie, des Privateigentums und des Scaats, MEW 21.25-173. - Zur Frage des Rechtsbegriffs in der Ethnologie gibt es unübersehbare Literatur, den besten Uberblick bei E. Adamson Hoebel, Das Reche der Naturvölker, 1968, S. 29-4I. Hoebel ist auch die besce Einführung in das Rechr der frühen Gescllschaften, er beschrcibt dic Ordnung der Eskimo, der Komantschen, Kiowa und Cheyenne, der berühmen Trobriander, die Malinowskj erforscht hat, und der Aschanti, allerdings rein funktional, niche historisch, William Seagle, Welıgeschichte des Rechis, 3. Auf1. 1967 , ist der einzige in neuer Zeit in deutscher Sprache erschienene Versuch, auch die Ethoologic in die Geschichte des Rechrs einzubeziehen, und zu Unrecht wird er ständig übersehen. Stanley Diamona', Kritik der Zivilisation, Anthropologie und die Wiederenteckung des Primitiven, 1976, bringt im 6. Kapitel die Beschreibung des Verhälınisses von Rechrsgesellschaften und Gewohnheitsgesellschaften. Pierre Clastres, Sraatsfeinde, 1976. - Die wichtigste Literatur zu Jägergesellschafren: Richard B. Lee, Irven DcVore, Man the Hunter, Chicago 1968, ein Sammelband, der die Engebnisse einer Tagung von 1965 zusammenfaßt und die bcște und erschöpfende Ubersicht über allc Probleme von Jägergesellschaften gibr. Die Forschung ist sehr stark becinflußr worden von Colin M. Tumbull, Wayward Servants, 1965, der das Prinzip der Fluktuazion bei den Mburi-Pygmäen enteckt har, und Marshall Sablins, Stone Age Economics, 1972. Die Guayaki sind beschrieben von Pierre Clastres, Chronique des indien Guayaki, 1972. - Zu den segmentären Gesellschafien: Die beste Ubersicht über die frühe Entwicklung in Mesopotamien in dem Buch des Ausgräbers von Catal Hüyük, James Mellaart, The Neolithic of the Near East, 1975. Sein Werk über die Ausgrabung ist auf Deutsch erschienen: James Mellaart, Casal Hüyük, Stadt aus der Steinzeit, 2. Aufl, 1967. Die Siedlungsstruktur mit der Verteilung der Kultstätten dort S. 67-77 und S. 83-89. Allgemeine Werke in deutscher Sprache zu segmentären Gesellschaften: Christian Sigrist, Regulierte Anarchie, Untersuchungen zum Fehlen und zur Entstehung politischer Herrschaft in segmentären Gesellschaiten Afrikas, 1967, und Fritz Kramer, Christian Sigrist (Hg.), Gesellschaften ohne Staat, 2 Bände, 1978 und 1979. Die Nuer beschreibe E. E. Evans-Pritchard, The Nuer, 1940, die Tallensi Meyer Forzes, The dynamics of clanship among the Tallensi, 1945, und The web of kinship among the Tallensi, 2. Aufl. 1957, die Lele Mary Dougles, The Lele of the Kasai, 1963. Alle drei Gesellschafien sind, unter anderem auch die Mburi-Jäger, jeweils einzeln sehr gut charakterisiert in dem Anfängerlehrbuch von Lucy Mair, African Sociecies, 1976. - Zum Matriarchat und zur Matrilinearität: Claude Meillassoux, Die wilden Früchte der Frau, 1976, der deutsche Titel ist unglücklich ubersetzt, der Iranzösische: Femmes, greniers cr capitaux, 1975. Neuere amerikanische Forschungen: David M. Schneider, Karhleen Gough (Hg.), Marrilineal Kinship, 196r, Alice Schlegel, Male dominance and female autonomy, 1972, Michelle Z. Rosaldo, Louise Lamphere ( $\mathrm{Hg}$.$) , Woman, Culcure and Sociery, 1974, Rayna R.$ Reiter (Hg.), Toward an Anthropology of Woman, r975. - Zur Entstehung von Herrschaft hat sich schon Sigrist 1967 geäußen und Clastres in den Statsfeinden. Beide sind oben genannt. Einen sehr guten Úberblick über die Geschichte der Lehrmeinungen und die Probleme gibe Elman R. Service, Ursprünge des Staates und der Zivilisation, 1977. Eine Auswahl der wichtigsten Schriften bei Klaws Eder ( $\mathrm{Hg}$.), Seminar: Die Entstehung von Klassengesellschaften, 1973. Zu Ankole: Kalervo Obero. The Kingdom of Ankole in Úganda, in: M. Fores, E. E. Evans-Pritchard ( $\mathrm{H}_{\mathrm{g}}$ ), African Political Systems, 1940, S. $12 \mathrm{I}-162$. Zu den Nupe: Siegfried F. Nadel, A Black Byzancium, r942. - Zur Gesellschaft und zum Recht der Aschanti gibe es die klassischen Werke von $A$. R. Rattray, Ashanti, 1923, und Ashanti, Law and Constitution, 1929. Die beste kurze Beschreibung bei Hoebcl, S. 26 $\{-319$. Die Arbeit von Busia: K. A. Busia, The position of the chief in the modern political system of Ashanti, I95r. 